\title{
A Class of Finsler Metrics with Isotropic S-curvature
}

\author{
Xinyue Cheng* and Zhongmin Shen ${ }^{\dagger}$
}

November 30, 2006

\begin{abstract}
In this paper, we study a class of Finsler metrics defined by a Riemannian metric and a 1 -form. We characterize these metrics with isotropic S-curvature.
\end{abstract}

\section{Introduction}

The S-curvature is one of the most important non-Riemannian quantities in Finsler geometry which was first introduced by the second author when he studied volume comparison in Riemann-Finsler geometry [10]. The second author proved that the Bishop-Gromov volume comparison holds for Finsler manifolds with vanishing S-curvature. He also proved that the S-curvature and the Ricci curvature determine the local behavior of the Busemann-Hausdorff measure of small metric balls around a point [16]. Recent study shows that the S-curvature plays a very important role in Finsler geometry (cf. [8][12][15]). It is known that, for a Finsler metric $F$ of scalar flag curvature, if the S-curvature is almost isotropic, i.e.,

$$
\mathbf{S}=(n+1) c F+\eta,
$$

where $c=c(x)$ is a scalar function and $\eta$ is a closed 1-form, then the flag curvature must be in the following form

$$
\mathbf{K}=\frac{3 \tilde{c}_{x^{m}} y^{m}}{F}+\sigma,
$$

where $\sigma=\sigma(x)$ and $\tilde{c}=\tilde{c}(x)$ are scalar functions with $c-\tilde{c}=$ constant [4]. Therefore it is an important problem to study and characterize Finsler metrics of (almost) isotropic S-curvature.

In Finsler geometry, there is an important class of Finsler metrics-Randers metrics which were introduced and studied by G. Randers. A Randers metric

\footnotetext{
* Supported by the National Natural Science Foundation of China(10671214) and by Natural Science Foundation Project of CQ CSTC

${ }^{\dagger}$ Supported by the National Natural Science Foundation of China(10671214) and by a NSF grant on IR/D
} 
is a Finsler metric expressed in the form $F=\alpha+\beta$, where $\alpha=\sqrt{a_{i j}(x) y^{i} y^{j}}$ is a Riemannian metric and $\beta=b_{i}(x) y^{i}$ is a 1 -form with $\left\|\beta_{x}\right\|_{\alpha}<1$. Let

$$
\begin{gathered}
r_{i j}=\frac{1}{2}\left(b_{i \mid j}+b_{j \mid i}\right), \quad s_{i j}=\frac{1}{2}\left(b_{i \mid j}-b_{j \mid i}\right), \\
r_{j}:=b^{i} r_{i j}, \quad s_{j}:=b^{i} s_{i j},
\end{gathered}
$$

where $b_{i \mid j}$ denote the covariant derivatives of $\beta$ with respect to $\alpha$. In [5], we prove that the Randers metric $F=\alpha+\beta$ has isotropic S-curvature, $\mathbf{S}=(n+1) c(x) F$, if and only if

$$
r_{i j}+b_{i} s_{j}+b_{j} s_{i}=2 c\left(a_{i j}-b_{i} b_{j}\right) .
$$

See [1] [17] for related work. In this paper, we generalize the above result as follows.

\section{Theorem 1.1 Let}

$$
F=k_{1} \sqrt{\alpha^{2}+k_{2} \beta^{2}}+k_{3} \beta
$$

be a Finsler metric of Randers type where $k_{1}>0$ and $k_{3} \neq 0 . F$ is of isotropic $S$-curvature, $F=(n+1) c F$ if and only if $\beta$ satisfies

$$
r_{i j}+\tau\left(s_{i} b_{j}+s_{j} b_{i}\right)=\frac{2 c\left(1+k_{2} b^{2}\right) k_{1}^{2}}{k_{3}}\left(a_{i j}-\tau b_{i} b_{j}\right),
$$

where

$$
\tau:=\frac{k_{3}^{2}}{k_{1}^{2}}-k_{2}
$$

If a Randers metric is of scalar flag curvature, then (1) and (2) are actually equivalent ([7], [18]). In particular, if a Randers metric is of constant flag curvature, then it must be of constant S-curvature ([1], [2]). We have classified Randers metrics of scalar flag curvature and isotropic S-curvature ([4], [7]). Further, we have characterized the locally projectively flat Finsler metrics with isotropic S-curvature ([6]).

It is natural to consider general Finsler metrics defined by a Riemannian metric $\alpha=\sqrt{a_{i j} y^{i} y^{j}}$ and a 1-form $\beta=b_{i} y^{i}$ with $\left\|\beta_{x}\right\|_{\alpha}<b_{o}$. They are expressed in the form $F=\alpha \phi(s), s=\beta / \alpha$, where $\phi(s)$ is a $C^{\infty}$ positive function on $\left(-b_{o}, b_{o}\right)$. It is known that $F=\alpha \phi(\beta / \alpha)$ is a (positive definite) Finsler metric for any $\alpha$ and $\beta$ with $\left\|\beta_{x}\right\|_{\alpha}<b_{o}$ if and only if $\phi$ satisfies the following condition (cf. [13][14]):

$$
\phi(s)-s \phi^{\prime}(s)+\left(\rho^{2}-s^{2}\right) \phi^{\prime \prime}(s)>0, \quad\left(|s| \leq \rho<b_{o}\right) .
$$

Such a metric is called an $(\alpha, \beta)$-metric. Clearly, Finsler metrics of Randers type are special $(\alpha, \beta)$-metrics. let

For a positive $C^{\infty}$ function $\phi=\phi(s)$ on $\left(-b_{o}, b_{o}\right)$ and a number $b \in\left[0, b_{o}\right)$,

$$
\Phi:=-\left(Q-s Q^{\prime}\right)\{n \Delta+1+s Q\}-\left(b^{2}-s^{2}\right)(1+s Q) Q^{\prime \prime},
$$

where $\Delta:=1+s Q+\left(b^{2}-s^{2}\right) Q^{\prime}$ and $Q:=\phi^{\prime} /\left(\phi-s \phi^{\prime}\right)$. In this paper, we prove the following 
Theorem 1.2 Let $F=\alpha \phi(s), s=\beta / \alpha$, be an $(\alpha, \beta)$-metric on a manifold and $b:=\left\|\beta_{x}\right\|_{\alpha}$. Suppose that $\phi \neq k_{1} \sqrt{1+k_{2} s^{2}}+k_{3} s$ for any constants $k_{1}>0, k_{2}$ and $k_{3}$. Then $F$ is of isotropic $S$-curvature, $\mathbf{S}=(n+1) c F$, if and only if one of the following holds

(i) $\beta$ satisfies

$$
r_{j}+s_{j}=0
$$

and $\phi=\phi(s)$ satisfies

$$
\Phi=0
$$

In this case, $\mathbf{S}=0$.

(ii) $\beta$ satisfies

$$
r_{i j}=\epsilon\left\{b^{2} a_{i j}-b_{i} b_{j}\right\}, \quad s_{j}=0,
$$

where $\epsilon=\epsilon(x)$ is a scalar function, and $\phi=\phi(s)$ satisfies

$$
\Phi=-2(n+1) k \frac{\phi \Delta^{2}}{b^{2}-s^{2}},
$$

where $k$ is a constant. In this case, $\mathbf{S}=(n+1) c F$ with $c=k \epsilon$.

(iii) $\beta$ satisfies

$$
r_{i j}=0, \quad s_{j}=0 .
$$

In this case, $\mathbf{S}=0$, regardless of the choice of a particular $\phi$.

It is easy to see that (10) implies (8), while (8) implies (6). The condition (6) is equivalent to that $b:=\left\|\beta_{x}\right\|_{\alpha}=$ constant. See Lemma 3.2 below. Thus (7) and (9) are independent of $x \in M$.

The mean Landsberg curvature $\mathbf{J}$ is another important non-Riemannian quantity. It has been proved that for an $(\alpha, \beta)$-metric $F=\alpha \phi(\beta / \alpha)$, if $\beta$ has constant length and $\phi$ satisfies (7), then $F$ is a weakly Landsberg metric, i.e., $\mathbf{J}=0$. See [9].

We have the following two interesting examples.

Example 1.1 Let $F=\alpha+\beta$ be the family of Randers metrics on $S^{3}$ constructed in [3] (see also [16]). It is shown that $r_{i j}=0$ and $s_{j}=0$. Thus for any $C^{\infty}$ positive function $\phi=\phi(s)$ satisfying (5), the $(\alpha, \beta)$-metric $F=\alpha \phi(\beta / \alpha)$ has vanishing S-curvature.

Example 1.2 Let $F=\alpha \phi(\beta / \alpha)$ be an $(\alpha, \beta)$-metric defined on an open subset in $R^{3}$. At a point $\mathbf{x}=(x, y, z) \in R^{3}$ and in the direction $\mathbf{y}=(u, v, w) \in T_{\mathbf{x}} R^{3}$, $\alpha=\alpha(\mathbf{x}, \mathbf{y})$ and $\beta=\beta(\mathbf{x}, \mathbf{y})$ are given by

$$
\begin{aligned}
\alpha & :=\sqrt{u^{2}+e^{2 x}\left(v^{2}+w^{2}\right)}, \\
\beta & :=u .
\end{aligned}
$$

Then $\beta$ satisfies (8) with $\epsilon=1, b=1$. Thus if $\phi=\phi(s)$ satisfies (9) for some constant $k$, then $F=\alpha \phi(\beta / \alpha)$ is of constant S-curvature $\mathbf{S}=(n+1) c F$. 


\section{Volume forms}

The S-curvature is associated with a volume form. There are two important volume forms in Finsler geometry. One is the Busemann-Hausdorff volume form and the other is the Holmes-Thompson volume form.

The Busemann-Hausdorff volume form $d V_{B H}=\sigma_{B H}(x) d x$ is given by

$$
\sigma_{B H}(x)=\frac{\omega_{n}}{\operatorname{Vol}\left\{\left(y^{i}\right) \in R^{n} \mid F\left(x, y^{i} \frac{\partial}{\partial x^{i}}\right)<1\right\}}
$$

and the Holmes-Thompson volume form $d V_{H T}=\sigma_{H T}(x) d x$ is given by

$$
\sigma_{H T}(x)=\frac{1}{\omega_{n}} \int_{\left\{\left(y^{i}\right) \in R^{n} \mid F\left(x, y^{i} \frac{\partial}{\partial x^{i}}\right)<1\right\}} \operatorname{det}\left(g_{i j}\right) d y .
$$

Here Vol denotes the Euclidean volume and

$$
\omega_{n}:=\operatorname{Vol}\left(\mathrm{B}^{n}(1)\right)=\frac{1}{n} \operatorname{Vol}\left(\mathrm{S}^{n-1}\right)=\frac{1}{n} \operatorname{Vol}\left(\mathrm{S}^{n-2}\right) \int_{0}^{\pi} \sin ^{n-2}(t) d t
$$

denotes the Euclidean volume of the unit ball in $R^{n}$. When $F=\sqrt{g_{i j}(x) y^{i} y^{j}}$ is a Riemannian metric, both volume forms are reduced to the same Riemannian volume form

$$
d V_{B H}=d V_{H T}=\sqrt{\operatorname{det}\left(g_{i j}(x)\right)} d x .
$$

For an $(\alpha, \beta)$-metric, we have the following formulas for the volume forms $d V_{B H}$ and $d V_{H T}$.

Proposition 2.1 Let $F=\alpha \phi(s), s=\beta / \alpha$, be an $(\alpha, \beta)$-metric on an n-dimensional manifold $M$. Let $d V=d V_{B H}$ or $d V_{H T}$. Let

$$
f(b):= \begin{cases}\frac{\int_{0}^{\pi} \sin ^{n-2} t d t}{\int_{0}^{\pi} \frac{\sin ^{n-2} t}{\phi(b \cos t)^{n}} d t} & \text { if } d V=d V_{B H} \\ \frac{\int_{0}^{\pi}\left(\sin ^{n-2} t\right) T(b \cos t) d t}{\int_{0}^{\pi} \sin ^{n-2} t d t} & \text { if } d V=d V_{T H},\end{cases}
$$

where $T(s):=\phi\left(\phi-s \phi^{\prime}\right)^{n-2}\left[\left(\phi-s \phi^{\prime}\right)+\left(b^{2}-s^{2}\right) \phi^{\prime \prime}\right]$. Then the volume form $d V$ is given by

$$
d V=f(b) d V_{\alpha},
$$

where $d V_{\alpha}=\sqrt{\operatorname{det}\left(a_{i j}\right)} d x$ denotes the Riemannian volume form of $\alpha$.

Proof: In a coordinate system, the determinant of $g_{i j}:=\frac{1}{2}\left[F^{2}\right]_{y^{i} y^{j}}$ is given by (cf. [14])

$$
\operatorname{det}\left(g_{i j}\right)=\phi^{n+1}\left(\phi-s \phi^{\prime}\right)^{n-2}\left[\left(\phi-s \phi^{\prime}\right)+\left(b^{2}-s^{2}\right) \phi^{\prime \prime}\right] \operatorname{det}\left(a_{i j}\right) .
$$

First we take an orthonormal basis at $x$ with respect to $\alpha$ so that

$$
\alpha=\sqrt{\sum\left(y^{i}\right)^{2}}, \quad \beta=b y^{1},
$$


where $b=\left\|\beta_{x}\right\|_{\alpha}$. Then the volume form $d V_{\alpha}=\sigma_{\alpha} d x$ at $x$ is given by

$$
\sigma_{\alpha}=\sqrt{\operatorname{det}\left(a_{i j}\right)}=1
$$

In order to evaluate the integrals

$$
\operatorname{Vol}\left\{\left(y^{i}\right) \in R^{n} \mid F\left(x, y^{i} \frac{\partial}{\partial x^{i}}\right)<1\right\}=\int_{F(x, y)<1} d y=\int_{\alpha \phi(\beta / \alpha)<1} d y
$$

and

$$
\int_{F(x, y)<1} \operatorname{det}\left(g_{i j}\right) d y=\int_{\alpha \phi(\beta / \alpha)<1} \operatorname{det}\left(g_{i j}\right) d y,
$$

we take the following coordinate transformation, $\psi:\left(s, u^{a}\right) \rightarrow\left(y^{i}\right)$ :

$$
y^{1}=\frac{s}{\sqrt{b^{2}-s^{2}}} \bar{\alpha}, \quad y^{a}=u^{a},
$$

where $\bar{\alpha}=\sqrt{\sum_{a=2}^{n}\left(u^{a}\right)^{2}}$. Then

$$
\alpha=\frac{b}{\sqrt{b^{2}-s^{2}}} \bar{\alpha}, \quad \beta=\frac{b s}{\sqrt{b^{2}-s^{2}}} \bar{\alpha} .
$$

Thus

$$
F=\alpha \phi(\beta / \alpha)=\frac{b \phi(s)}{\sqrt{b^{2}-s^{2}}} \bar{\alpha}
$$

and the Jacobian of the transformation $\psi:\left(s, u^{a}\right) \rightarrow\left(y^{i}\right)$ is given by

$$
\frac{b^{2}}{\left(b^{2}-s^{2}\right)^{3 / 2}} \bar{\alpha} \text {. }
$$

Then

$$
\begin{aligned}
\operatorname{Vol}\left\{\left(y^{i}\right) \in R^{n} \mid F(x, y)<1\right\} & =\int_{\frac{b \phi(s)}{\sqrt{b^{2}-s^{2}}} \bar{\alpha}<1} \frac{b^{2}}{\left(b^{2}-s^{2}\right)^{3 / 2}} \bar{\alpha} d s d u \\
& =\int_{-b}^{b} \frac{b^{2}}{\left(b^{2}-s^{2}\right)^{3 / 2}}\left[\int_{\bar{\alpha}<\frac{\sqrt{b^{2}-s^{2}}}{b \phi(s)}} \bar{\alpha} d u\right] d s \\
& =\frac{1}{n} \operatorname{Vol}\left(\mathrm{S}^{n-2}\right) \int_{-b}^{b} \frac{b^{2}}{\left(b^{2}-s^{2}\right)^{3 / 2}}\left(\frac{\sqrt{b^{2}-s^{2}}}{b \phi(s)}\right)^{n} d s \\
& =\frac{1}{n} \operatorname{Vol}\left(\mathrm{S}^{n-2}\right) \int_{-b}^{b} \frac{\left(b^{2}-s^{2}\right)^{(n-3) / 2}}{b^{n-2} \phi(s)^{n}} d s \\
& =\frac{1}{n} \operatorname{Vol}\left(\mathrm{S}^{n-2}\right) \int_{0}^{\pi} \frac{\sin ^{n-2} t}{\phi(b \cos t)^{n}} d t \quad(s=b \cos t) .
\end{aligned}
$$

Therefore

$$
\sigma_{B H}=\frac{\int_{0}^{\pi} \sin ^{n-2} t d t}{\int_{0}^{\pi} \frac{\sin ^{n-2} t}{\phi(b \cos t)^{n}} d t} .
$$


Let

$$
T(s):=\phi\left(\phi-s \phi^{\prime}\right)^{n-2}\left[\left(\phi-s \phi^{\prime}\right)+\left(b^{2}-s^{2}\right) \phi^{\prime \prime}\right] .
$$

Then

$$
\operatorname{det}\left(g_{i j}\right)=\phi(s)^{n} T(s) \operatorname{det}\left(a_{i j}\right) .
$$

By a similar argument, we get

$$
\begin{aligned}
\sigma_{H T} & =\frac{1}{\omega_{n}} \int_{F(x, y)<1} \phi(s)^{n} T(s) d y^{1} \cdots d y^{n} \\
& =\frac{1}{n \omega_{n}} \operatorname{Vol}\left(\mathrm{S}^{n-2}\right) \int_{-b}^{b} \frac{b^{2}}{\left(b^{2}-s^{2}\right)^{3 / 2}}\left(\frac{\sqrt{b^{2}-s^{2}}}{b}\right)^{n} T(s) d s \\
& =\frac{\int_{0}^{\pi}\left(\sin ^{n-2} t\right) T(b \cos t) d t}{\int_{0}^{\pi} \sin ^{n-2} t d t} .
\end{aligned}
$$

Thus

$$
\sigma_{H T}=\frac{\int_{0}^{\pi}\left(\sin ^{n-2} t\right) T(b \cos t) d t}{\int_{0}^{\pi} \sin ^{n-2} t d t} .
$$

The above formulas for $\sigma_{B H}$ and $\sigma_{H T}$ are given in a special coordinate system at $x$ and $\sigma_{\alpha}=1$. Thus $d V=f(b) d V_{\alpha}$. This proves the proposition. Q.E.D.

Note that if $b=$ constant, then $f(b)=$ constant. In this case, both $d V_{B H}$ and $d V_{H T}$ are constant multiples of $d V_{\alpha}$.

It is surprised to see that $d V_{T H}=d V_{\alpha}$ for certain functions $\phi$.

Corollary 2.2 Let $F=\alpha \phi(s), s=\beta / \alpha$, be an $(\alpha, \beta)$-metric on an $n$-dimensional manifold $M$. Let $T=T(s)$ be defined in (13). Suppose that $T(s)-1$ is an odd function of $s$. Then $d V_{T H}=d V_{\alpha}$.

Proof: Let $h(s)=T(s)-1$. By assumption $h(-s)=-h(s)$. It is easy to see that

$$
\int_{0}^{\pi}\left(\sin ^{n-2} t\right) h(b \cos t) d t=0 .
$$

Thus

$$
\int_{0}^{\pi}\left(\sin ^{n-2} t\right) T(b \cos t) d t=\int_{0}^{\pi} \sin ^{n-2} t d t .
$$

This implies that $\sigma_{H T}=1$ in the above special coordinate system at $x$. Then in a general coordinate system $\sigma_{H T}=\sigma_{\alpha}$.

Q.E.D.

If $\phi=1+s$, then $T=1+s$ and $T(s)-1$ is an odd function of $s$. Then for a Randers metric, $d V_{H T}=d V_{\alpha}$. This fact is known to Y. B. Shen. 


\section{The S-Curvature}

In this section, we are going to find a formula for the S-curvature of an $(\alpha, \beta)$ metric on an $n$-dimensional manifold $M$.

Let $F=F(x, y)$ be a Finsler metric on an $n$-dimensional manifold $M$. Let $G=y^{i} \frac{\partial}{\partial x^{i}}-2 G^{i} \frac{\partial}{\partial y^{i}}$ denote the spray of $F$ and $d V=\sigma d x$ be a volume form on $M$. The spray coefficients $G^{i}$ are defined by

$$
G^{i}=\frac{1}{4} g^{i l}\left\{\left[F^{2}\right]_{x^{j} y^{l}} y^{j}-\left[F^{2}\right]_{x^{l}}\right\} .
$$

Then the S-curvature (with respect to $d V$ ) is defined by

$$
\mathbf{S}=\frac{\partial G^{m}}{\partial y^{m}}-y^{m} \frac{\partial}{\partial x^{m}}(\ln \sigma)
$$

By the definition, S-curvature $\mathbf{S}(y)$ measures the average rate of changes of $\left(T_{x} M, F_{x}\right)$ in the direction $y \in T_{x} M$. An important property is that $\mathbf{S}=0$ for Berwald spaces with respect to the Busemann-Hausdorff volume form $d V_{B H}$ [10][11].

Definition 3.1 Let $F$ be a Finsler metric on an $n$-dimensional manifold $M$. Let $\mathbf{S}$ denote the S-curvature of $F$ with respect to $d V_{B H} . \quad F$ is of isotropic S-curvature if

$$
\mathbf{S}=(n+1) c F
$$

where $c=c(x)$ is a scalar function. $F$ is of constant S-curvature if $c=$ constant.

We now compute the S-curvature of an $(\alpha, \beta)$-metric on a manifold. Let

$$
F=\alpha \phi(s), \quad s=\beta / \alpha .
$$

We have the following formula for the spray coefficients $G^{i}$ of $F$ :

$$
G^{i}=\bar{G}^{i}+\alpha Q s_{0}^{i}+\Theta\left\{-2 Q \alpha s_{0}+r_{00}\right\} \frac{y^{i}}{\alpha}+\Psi\left\{-2 Q \alpha s_{0}+r_{00}\right\} b^{i},
$$

where $\bar{G}^{i}$ denote the spray coefficients of $\alpha$ and

$$
Q:=\frac{\phi^{\prime}}{\phi-s \phi^{\prime}}, \quad \Theta=\frac{Q-s Q^{\prime}}{2 \Delta}, \quad \Psi=\frac{Q^{\prime}}{2 \Delta},
$$

where $\Delta:=1+s Q+\left(b^{2}-s^{2}\right) Q^{\prime}$.

It is easy to see that if $\phi=\phi(s)$ satisfies

$$
b^{2} Q+s=0,
$$

then

$$
\phi=a_{0} \sqrt{b^{2}-s^{2}}
$$


where $a_{0}$ is a number independent of $s$.

If $\phi=\phi(s)$ satisfies

$$
\Psi=\text { constant }
$$

then

$$
\phi=k_{1} \sqrt{1+k_{2} s^{2}}+k_{3} s,
$$

where $k_{1}, k_{2}$ and $k_{3}$ are numbers independent of $s$.

To compute the S-curvature, we need the following identities:

$$
\begin{gathered}
\frac{\partial s}{\partial y^{m}}=\frac{1}{\alpha}\left\{b_{m}-s \frac{y_{m}}{\alpha}\right\}, \\
\frac{\partial \alpha}{\partial y^{m}}=\frac{y_{m}}{\alpha}, \\
\frac{\partial \bar{G}^{m}}{\partial y^{m}}=y^{m} \frac{\partial}{\partial x^{m}}\left(\ln \sigma_{\alpha}\right) .
\end{gathered}
$$

Using the above identities, we obtain

$$
\frac{\partial G^{m}}{\partial y^{m}}=y^{m} \frac{\partial}{\partial x^{m}}\left(\ln \sigma_{\alpha}\right)+2 \Psi\left(r_{0}+s_{0}\right)-\alpha^{-1} \frac{\Phi}{2 \Delta^{2}}\left(r_{00}-2 \alpha Q s_{0}\right),
$$

where

$$
\Phi:=-\left(Q-s Q^{\prime}\right)\{n \Delta+1+s Q\}-\left(b^{2}-s^{2}\right)(1+s Q) Q^{\prime \prime} .
$$

By Proposition 2.1, $d V=\sigma d x=f(b) \sigma_{\alpha} d x$. Thus

$$
\begin{gathered}
y^{m} \frac{\partial}{\partial x^{m}}(\ln \sigma)=\frac{f^{\prime}(b)}{f(b)} y^{m} \frac{\partial b}{\partial x^{m}}+y^{m} \frac{\partial}{\partial x^{m}}\left(\ln \sigma_{\alpha}\right) . \\
y^{m} \frac{\partial b}{\partial x^{m}}=\frac{b^{i} b_{i \mid m} y^{m}}{b}=\frac{r_{0}+s_{0}}{b} .
\end{gathered}
$$

Then the S-curvature is given by

$$
\mathbf{S}=\left\{2 \Psi-\frac{f^{\prime}(b)}{b f(b)}\right\}\left(r_{0}+s_{0}\right)-\alpha^{-1} \frac{\Phi}{2 \Delta^{2}}\left(r_{00}-2 \alpha Q s_{0}\right) .
$$

Lemma 3.2 Let $\beta$ be a 1-form on a Riemannian manifold $(M, \alpha)$. Then $b(x):=$ $\left\|\beta_{x}\right\|_{\alpha}=$ constnt if and only if $\beta$ satisfies the following equation:

$$
r_{j}+s_{j}=0 .
$$

Proof: This follows immediately from (17).

Q.E.D.

In the case when $b=$ constant, the S-curvature is given by

$$
\mathbf{S}=-\alpha^{-1} \frac{\Phi}{2 \Delta^{2}}\left(r_{00}-2 \alpha Q s_{0}\right) .
$$

We can prove the following 
Proposition 3.3 Let $F=\alpha \phi(\beta / \alpha)$ be an $(\alpha, \beta)$-metric on an $n$-manifold. If $\beta$ and $\phi$ satisfy conditions in Theorem 1.2 (i) or (ii) or (iii), then $F$ has isotropic S-curvature.

Proof: If $\beta$ satisfies (6) and $\phi$ satisfies (7), then it follows from (18) that $\mathbf{S}=0$.

If $\beta$ satisfies (8), then

$$
r_{00}=\epsilon\left(b^{2}-s^{2}\right) \alpha^{2}, \quad r_{0}=0, \quad s_{0}=0 .
$$

By (9) and the above equations, we get from (18) that

$$
\mathbf{S}=-\alpha \epsilon\left(b^{2}-s^{2}\right) \frac{\Phi}{2 \Delta^{2}}=(n+1) k \epsilon \alpha \phi=(n+1) k \epsilon F .
$$

If $\beta$ satisfies (10), then

$$
r_{00}=0, \quad r_{0}=0, \quad s_{0}=0 .
$$

It follows from (18) that $\mathbf{S}=0$.

Q.E.D.

To prove the necessary conditions in Theorem 1.2, we consider an $(\alpha, \beta)$ metric $F=\alpha \phi(\beta / \alpha)$ with isotropic S-curvature, $\mathbf{S}=(n+1) c F$. By (18), the equation $\mathbf{S}=(n+1) c F$ is equivalent to the following equation:

$$
\alpha^{-1} \frac{\Phi}{2 \Delta^{2}}\left(r_{00}-2 \alpha Q s_{0}\right)-2 \Psi\left(r_{0}+s_{0}\right)=-(n+1) c F+\theta,
$$

where

$$
\theta:=-\frac{f^{\prime}(b)}{b f(b)}\left(r_{0}+s_{0}\right)
$$

To simplify the equation (21), we choose special coordinates. Fix an arbitrary point $x$. Take a local coordinate system at $x$ as in (11). We have

$$
\begin{gathered}
r_{1}=b r_{11}, \quad r_{\alpha}=b r_{1 \alpha}, \\
s_{1}=0, \quad s_{\alpha}=b s_{1 \alpha} .
\end{gathered}
$$

Let

$$
\begin{gathered}
\bar{r}_{10}:=\sum_{\alpha=2}^{n} r_{1 \alpha} y^{\alpha}, \quad \bar{s}_{10}:=\sum_{\alpha=2}^{n} s_{1 \alpha} y^{\alpha} \quad \bar{r}_{00}:=\sum_{\alpha, \beta=2}^{n} r_{\alpha \beta} y^{\alpha} y^{\beta}, \\
\bar{r}_{0}:=\sum_{\alpha=2}^{n} r_{\alpha} y^{\alpha} \quad \bar{s}_{0}:=\sum_{\alpha=2}^{n} s_{\alpha} y^{\alpha} .
\end{gathered}
$$

We have

$$
\bar{r}_{0}=b \bar{r}_{10}, \quad \bar{s}_{0}=b \bar{s}_{10} .
$$

Let $\theta=t_{i} y^{i}$. Then $t_{i}$ are given by

$$
t_{1}=-\frac{f^{\prime}(b)}{f(b)} r_{11}, \quad t_{\alpha}=-\frac{f^{\prime}(b)}{f(b)}\left(r_{1 \alpha}+s_{1 \alpha}\right) .
$$


(21) is equivalent to the following two equations:

$$
\begin{gathered}
\frac{\Phi}{2 \Delta^{2}}\left(b^{2}-s^{2}\right) \bar{r}_{00}=-\left\{s\left(\frac{s \Phi}{2 \Delta^{2}}-2 \Psi b^{2}\right) r_{11}+(n+1) c b^{2} \phi-s b t_{1}\right\} \bar{\alpha}^{2}, \\
\left(\frac{s \Phi}{\Delta^{2}}-2 \Psi b^{2}\right)\left(r_{1 \alpha}+s_{1 \alpha}\right)-\left(b^{2} Q+s\right) \frac{\Phi}{\Delta^{2}} s_{1 \alpha}-b t_{\alpha}=0 .
\end{gathered}
$$

Let

$$
\Upsilon:=\left[\frac{s \Phi}{\Delta^{2}}-2 \Psi b^{2}\right]^{\prime} .
$$

We see that $\Upsilon=0$ if and only if

$$
\frac{s \Phi}{\Delta^{2}}-2 \Psi b^{2}=b^{2} \mu,
$$

where $\mu$ is a number independent of $s$. We shall divide the problem into three cases: (i) $\Phi=0$, (ii) $\Phi \neq 0, \Upsilon=0$ and (iii) $\Phi \neq 0, \Upsilon \neq 0$.

\section{$4 \Phi=0$}

In this section, we study the simplest case when $\Phi=0$.

Proposition 4.1 Let $F=\alpha \phi(\beta / \alpha)$ be an $(\alpha, \beta)$-metric. Assume that $\Phi=0$ but $\phi \neq k_{1} \sqrt{1+k_{2} s^{2}}$ for any constants $k_{1}>0$ and $k_{2}$. If $F$ has isotropic $S$-curvature, then

$$
r_{0}+s_{0}=0 .
$$

In this case, $\mathbf{S}=0$.

Proof: Take a special coordinate system at $x$ as in (11). (24) and (25) are reduced to

$$
\begin{gathered}
s\left\{\frac{f^{\prime}(b)}{b f(b)}-2 \Psi\right\} b^{2} r_{11}+(n+1) c b^{2} \phi=0 \\
\left\{\frac{f^{\prime}(b)}{b f(b)}-2 \Psi\right\} b^{2}\left(r_{1 \alpha}+s_{1 \alpha}\right)=0 .
\end{gathered}
$$

Letting $s=0$ in (26) yields

$$
c=0
$$

and

$$
\left\{\frac{f^{\prime}(b)}{b f(b)}-2 \Psi\right\} r_{11}=0 .
$$

If

$$
\frac{f^{\prime}(b)}{b f(b)}-2 \Psi=0,
$$

then

$$
\phi=k_{1} \sqrt{1+k_{2} s^{2}}+k_{3} s,
$$


where $k_{1}>0, k_{2}$ and $k_{3}$ are numbers independent of $s$. Plugging it into the equation $\Phi=0$ yields that $k_{3}=0$ and

$$
\phi=k_{1} \sqrt{1+k_{2} s^{2}} .
$$

But this is impossible by assumption. Thus

$$
\frac{f^{\prime}(b)}{b f(b)}-2 \Psi \neq 0 \text {. }
$$

From (26) and (27), we conclude that

$$
r_{11}=0, \quad r_{1 \alpha}+s_{1 \alpha}=0 .
$$

Q.E.D.

\section{$5 \Phi \neq 0, \Upsilon=0$}

First, note that $\Upsilon=0$ implies that

$$
\frac{s \Phi}{\Delta^{2}}-2 \Psi b^{2}=b^{2} \mu,
$$

where $\mu$ is a number independent of $s$. First, we prove the following

Lemma 5.1 Let $F=\alpha \phi(\beta / \alpha)$ be an $(\alpha, \beta)$-metric. Assume that $\Phi \neq 0$ and $\Upsilon=0$. If $F$ has isotropic $S$-curvature, $\mathbf{S}=(n+1) c F$, then $\beta$ satisfies

$$
r_{i j}=k a_{i j}-\epsilon b_{i} b_{j}+\frac{1}{b^{2}}\left(r_{i} b_{j}+r_{j} b_{i}\right),
$$

where $k=k(x), \epsilon=\epsilon(x)$, and $\phi=\phi(s)$ satisfies the following ODE:

$$
\left(k-\epsilon s^{2}\right) \frac{\Phi}{2 \Delta^{2}}=\left\{\nu+\left(k-\epsilon b^{2}\right) \mu\right\} s-(n+1) c \phi,
$$

where $\nu=\nu(x)$. If $s_{0} \neq 0$, then $\phi$ satisfies the following additional ODE:

$$
\frac{\Phi}{\Delta^{2}}\left(Q b^{2}+s\right)=b^{2}(\mu+\lambda)
$$

where $\lambda=\lambda(x)$.

Proof: Since $\Phi \neq 0$, it follows from (24) and (25) that in a special coordinate system $\left(s, y^{a}\right)$ at a point $x$,

$$
\begin{gathered}
r_{a b}=k \delta_{a b} \\
s\left(\frac{s \Phi}{2 \Delta^{2}}-2 \Psi b^{2}\right) r_{11}+(n+1) c b^{2} \phi+k \frac{\Phi}{2 \Delta^{2}}\left(b^{2}-s^{2}\right)=b s t_{1} \\
\left(\frac{s \Phi}{\Delta^{2}}-2 \Psi b^{2}\right)\left(r_{1 \alpha}+s_{1 \alpha}\right)-\left(b^{2} Q+s\right) \frac{\Phi}{\Delta^{2}} s_{1 \alpha}-b t_{\alpha}=0 .
\end{gathered}
$$


Let

$$
r_{11}=-\left(k-\epsilon b^{2}\right) .
$$

Then (30) holds. By (29), we have

$$
\frac{s \Phi}{2 \Delta^{2}}-2 \Psi b^{2}=b^{2} \mu-\frac{s \Phi}{2 \Delta^{2}} .
$$

Then (34) and (35) become

$$
\begin{gathered}
b\left(k-\epsilon s^{2}\right) \frac{\Phi}{2 \Delta^{2}}=s t_{1}+s b \mu\left(k-b^{2} \epsilon\right)-(n+1) c b \phi . \\
b^{2} \mu\left(r_{1 \alpha}+s_{1 \alpha}\right)-\frac{\Phi}{\Delta^{2}}\left(Q b^{2}+s\right) s_{1 \alpha}-b t_{\alpha}=0 .
\end{gathered}
$$

Letting $t_{1}=b \nu$ in (36) we get (31).

Suppose that $s_{0} \neq 0$. Rewrite (37) as

$$
\left\{b^{2} \mu-\frac{\Phi}{\Delta^{2}}\left(Q b^{2}+s\right)\right\} s_{1 \alpha}=b t_{a}-b^{2} \mu r_{1 \alpha}
$$

We can see that there is a number $\lambda$ such that

$$
\mu b^{2}-\frac{\Phi}{\Delta^{2}}\left(Q b^{2}+s\right)=-b^{2} \lambda .
$$

This gives (32).

Q.E.D.

Lemma 5.2 Let $F=\alpha \phi(\beta / \alpha)$ be an $(\alpha, \beta)$-metric. Assume that $\Upsilon=0$. Then $b=$ constant.

Proof: Suppose that $b \neq$ constant. Then $b$ can be viewed as a variable over the manifold. By assumption,

$$
\frac{s \Phi}{\Delta^{2}}-2 \Psi b^{2}=b^{2} \mu
$$

where $\mu=\mu(x)$. Note that $\Delta^{2}\left(\frac{s \Phi}{\Delta^{2}}-2 \Psi b^{2}-b^{2} \mu\right)$ is a polynomial of degree six in $b$ by (15). More precisely, we have

$$
-\mu Q^{\prime 2} b^{6}-\left\{Q^{\prime 2}-2 \mu Q^{\prime}\left(1+s Q-s^{2} Q^{\prime}\right)\right\} b^{4}+(\cdots) b^{2}+(\cdots)=0 .
$$

Thus

$$
\mu Q^{\prime 2}=0, \quad Q^{\prime 2}-2 \mu Q^{\prime}\left(1+s Q-s^{2} Q^{\prime}\right)=0 .
$$

Then $Q^{\prime}=0$, which implies that $\phi=1+c s$. This is impossible.

Q.E.D. 
Proposition 5.3 Let $F=\alpha \phi(\beta / \alpha)$ be an $(\alpha, \beta)$-metric. Suppose that $b^{2} Q+s \neq$ $0, \Phi \neq 0$ and $\Upsilon=0$. If $F$ has isotropic $S$-curvature, $\mathbf{S}=(n+1) c F$, then

$$
r_{i j}=\epsilon\left(b^{2} a_{i j}-b_{i} b_{j}\right), \quad s_{j}=0,
$$

where $\epsilon=\epsilon(x)$ is a scalar function, and $\phi=\phi(s)$ satisfies

$$
\epsilon\left(b^{2}-s^{2}\right) \Phi=-2(n+1) c \phi \Delta^{2} .
$$

If $\epsilon \neq 0$, then $c / \epsilon=$ constant.

Proof: First by Lemma 5.2 and Lemma 3.2, we have

$$
r_{0}+s_{0}=0 .
$$

Then by (18), we get

$$
\mathbf{S}=-\alpha^{-1} \frac{\Phi}{2 \Delta^{2}}\left\{r_{00}-2 \alpha Q s_{0}\right\}
$$

By Lemma 5.1,

$$
r_{00}=\left(k-\epsilon s^{2}\right) \alpha^{2}+\frac{2 s}{b^{2}} r_{0} \alpha
$$

Then

$$
\mathbf{S}=-\left(k-\epsilon s^{2}\right) \frac{\Phi}{2 \Delta^{2}} \alpha+\frac{\Phi}{b^{2} \Delta^{2}}\left(b^{2} Q+s\right) s_{0} .
$$

By (31), we get

$$
\mathbf{S}=-s\left\{\nu+\left(k-\epsilon b^{2}\right) \mu\right\} \alpha+\frac{\Phi}{b^{2} \Delta^{2}}\left(b^{2} Q+s\right) s_{0}+(n+1) c \phi \alpha .
$$

By our assumption, $\mathbf{S}=(n+1) c F$, we get from (41) that

$$
-s\left\{\nu+\left(k-\epsilon b^{2}\right) \mu\right\} \alpha+\frac{\Phi}{b^{2} \Delta^{2}}\left(b^{2} Q+s\right) s_{0}=0 .
$$

Letting $y^{i}=\delta b^{i}$ for a sufficiently small $\delta>0$ yields

$$
-\delta\left\{\nu+\left(k-\epsilon b^{2}\right) \mu\right\} b^{2}=0 .
$$

We conclude that

$$
\nu+\left(k-\epsilon b^{2}\right) \mu=0
$$

Then (42) is reduced to

$$
\frac{\Phi}{b^{2} \Delta^{2}}\left(b^{2} Q+s\right) s_{0}=0 .
$$

Since $\Phi \neq 0$ and $b^{2} Q+s \neq 0$, we conclude that

$$
s_{0}=0 .
$$


Then

$$
r_{0}=-s_{0}=0 .
$$

It follows from (30) that

$$
r_{i j}=k a_{i j}-\epsilon b_{i} b_{j} .
$$

Contracting (44) with $b^{i}$ gives

$$
r_{j}=\left(k-\epsilon b^{2}\right) b_{j}=0 .
$$

Since $\beta \neq 0$, we get

$$
k=\epsilon b^{2}
$$

and (44) becomes

$$
r_{i j}=\epsilon\left(b^{2} a_{i j}-b_{i} b_{j}\right) .
$$

Finally, (40) follows from (31), (43) and (45).

If $\epsilon \neq 0$, then letting $s=0$ in (40) yields that $c / \epsilon=$ constant since $b=$ constant.

Q.E.D.

\section{$6 \Phi \neq 0$ and $\Upsilon \neq 0$}

In this section, we shall consider the case when $\phi=\phi(s)$ satisfies

$$
\Phi \neq 0, \quad \Upsilon \neq 0 .
$$

First we need the following

Lemma 6.1 Let $F=\alpha \phi(s), s=\beta / \alpha$, be an $(\alpha, \beta)$-metric on an $n$-dimensional manifold. Assume that $\phi=\phi(s)$ satisfies (46). Suppose that $F$ has isotropic $S$-curvature, $\mathbf{S}=(n+1) c F$. Then

$$
r_{i j}=k a_{i j}-\epsilon b_{i} b_{j}-\lambda\left(s_{i} b_{j}+s_{j} b_{i}\right),
$$

where $\lambda=\lambda(x), k=k(x)$ and $\epsilon=\epsilon(x)$ are scalar functions of $x$ and

$$
-2 s\left(k-\epsilon b^{2}\right) \Psi+\left(k-\epsilon s^{2}\right) \frac{\Phi}{2 \Delta^{2}}+(n+1) c \phi-s \nu=0,
$$

where

$$
\nu:=-\frac{f^{\prime}(b)}{b f(b)}\left(k-\epsilon b^{2}\right) .
$$

If in addition $s_{0} \neq 0$, then

$$
-2 \Psi-\frac{Q \Phi}{\Delta^{2}}-\lambda\left(\frac{s \Phi}{\Delta^{2}}-2 \Psi b^{2}\right)=\delta,
$$

where

$$
\delta:=-\frac{f^{\prime}(b)}{b f(b)}\left(1-\lambda b^{2}\right)
$$


Proof: By assumption, $\Phi \not \equiv 0$. It follows from (24) that there is a number $k$ at $x$, independent of $s$, such that

$$
\begin{gathered}
\bar{r}_{00}=k \bar{\alpha}^{2} \\
s\left(\frac{s \Phi}{2 \Delta^{2}}-2 \Psi b^{2}\right) r_{11}+(n+1) c b^{2} \phi+k \frac{\Phi}{2 \Delta^{2}}\left(b^{2}-s^{2}\right)=s b t_{1} .
\end{gathered}
$$

Let

$$
r_{11}=k-\epsilon b^{2},
$$

where $\epsilon$ is a number independent of $s$. By $(23), t_{1}=b \nu$, where $\nu$ is given by (49). Plugging them into (53) yields (48).

Suppose that $s_{0}=0$. Then

$$
b s_{1 \alpha}=s_{\alpha}=0 .
$$

Then (25) is reduced to

$$
\left(\frac{s \Phi}{\Delta^{2}}-2 \Psi b^{2}\right) r_{1 \alpha}-b t_{\alpha}=0 .
$$

By assumption, $\Upsilon \neq 0$, we know that $\frac{s \Phi}{\Delta^{2}}-2 \Psi b^{2} \neq$ constant. It follows from (54) that

$$
r_{1 \alpha}=0, \quad t_{\alpha}=0 .
$$

The above identities together with $r_{11}=k-\epsilon b^{2}$ and $t_{1}=b \nu$ imply the following identities

$$
r_{i j}=k a_{i j}-\epsilon b_{i} b_{j}
$$

Suppose that $s_{0} \neq 0$. Then $s_{\alpha_{o}}=b s_{1 \alpha_{o}} \neq 0$ for some $\alpha_{o}$.

Differentiating (25) with respect to $s$ yields

$$
\Upsilon r_{1 \alpha}-\left[\frac{Q \Phi}{\Delta^{2}}+2 \Psi\right]^{\prime} b^{2} s_{1 \alpha}=0 .
$$

Let

$$
\lambda:=-\frac{r_{1 \alpha_{o}}}{b^{2} s_{1 \alpha_{o}}} .
$$

Plugging it into (56) yields

$$
-\lambda \Upsilon-\left[\frac{Q \Phi}{\Delta^{2}}+2 \Psi\right]^{\prime}=0 .
$$

It follows from (57) that

$$
\delta:=-\frac{Q \Phi}{\Delta^{2}}-2 \Psi-\lambda\left[\frac{s \Phi}{\Delta^{2}}-2 \Psi b^{2}\right]
$$


is a number independent of $s$. By assumption that $\Upsilon \neq 0$, we obtain from (56) and (57) that

$$
r_{1 \alpha}+\lambda b^{2} s_{1 \alpha}=0 .
$$

(52) and (58) together with $r_{11}=k-\epsilon b^{2}$ implies that

$$
r_{i j}+\lambda\left(b_{i} s_{j}+b_{j} s_{i}\right)=k a_{i j}-\epsilon b_{i} b_{j} .
$$

By (23) and (58),

$$
t_{\alpha}=\frac{f^{\prime}(b)}{f(b)}\left(b^{2} \lambda-1\right) s_{1 \alpha} .
$$

On the other hand, by (25) and (58), we obtain

$$
b t_{\alpha}=\delta b^{2} s_{1 \alpha} .
$$

Combining the above identities, we get (51).

Q.E.D.

Lemma 6.2 Let $F=\alpha \phi(s), s=\beta / \alpha$, be an $(\alpha, \beta)$-metric. Suppose that $\phi=$ $\phi(s)$ satisfies (46) and $\phi \neq k_{1} \sqrt{1+k_{2} s^{2}}+k_{3}$ s for any constants $k_{1}>0, k_{2}$ and $k_{3}$. If $F$ has isotropic $S$-curvature, then

$$
r_{j}+s_{j}=0 .
$$

Proof: Suppose that $r_{j}+s_{j} \neq 0$, then $b:=\left\|\beta_{x}\right\|_{\alpha} \neq$ constant in a neighborhood. We view $b$ as a variable in (48) and (50). Since $\phi=\phi(s)$ is a function independent of $x,(48)$ and (50) actually give rise infinitely many ODEs on $\phi$.

First, we consider (48). Let

$$
e q:=\Delta^{2}\left\{-2 s\left(k-\epsilon b^{2}\right) \Psi+\left(k-\epsilon s^{2}\right) \frac{\Phi}{2 \Delta^{2}}+(n+1) c \phi-s \nu\right\} .
$$

We have

$$
e q=\Xi_{0}+\Xi_{2} b^{2}+\Xi_{4} b^{4},
$$

where

$$
\Xi_{4}:=\{(\epsilon-\nu) s+(n+1) c \phi\} \frac{\phi^{2}}{\left(\phi-s \phi^{\prime}\right)^{4}}\left(\phi^{\prime \prime}\right)^{2} .
$$

It follows from (48) that $e q=0$. Thus

$$
\Xi_{0}=0, \quad \Xi_{2}=0, \quad \Xi_{4}=0 .
$$

Since $\phi^{\prime \prime} \neq 0$, the equation $\Xi_{4}=0$ is equivalent to the following Ode:

$$
(\epsilon-\nu) s+(n+1) c \phi=0 .
$$

we conclude that

$$
\epsilon=\nu, \quad c=0 .
$$


Then by a direct computation we get

$$
\Xi_{0}+\Xi_{2} s^{2}=-\frac{1}{2}(1+s Q)\left\{(n-1)\left(k-\epsilon s^{2}\right)\left(Q-s Q^{\prime}\right)+2 k Q+2 \epsilon s\right\} .
$$

Then $\Xi_{0}=0$ and $\Xi_{2}=0$ imply that

$$
(n-1)\left(k-\epsilon s^{2}\right)\left(Q-s Q^{\prime}\right)+2 k Q+2 \epsilon s=0,
$$

Suppose that $(k, \epsilon) \neq 0$. We claim that $k \neq 0$. If this is not true, i.e., $k=0$, then $\epsilon \neq 0$ and (60) is reduced to

$$
-(n-1) s\left(Q-s Q^{\prime}\right)+2=0 .
$$

Letting $s=0$, we get a contradiction.

Now we have that $k \neq 0$. It is easy to see that $Q(0)=0$. Let

$$
\tilde{Q}:=Q(s)-s Q^{\prime}(0) \text {. }
$$

Plugging it into (60) yields

$$
(n-1)\left(k-\epsilon s^{2}\right)\left(\tilde{Q}-s \tilde{Q}^{\prime}\right)+2 k \tilde{Q}+2\left(k Q^{\prime}(0)+\epsilon\right) s=0 .
$$

Since $\tilde{Q}=q_{m} s^{m}+o\left(s^{m}\right)$ where $m>1$ is an integer, we see that $k Q^{\prime}(0)+\epsilon=0$. The above equation is reduced to

$$
(n-1)\left(k-\epsilon s^{2}\right)\left(\tilde{Q}-s \tilde{Q}^{\prime}\right)+2 k \tilde{Q}=0 .
$$

We obtain

$$
\tilde{Q}=c_{1} \frac{s^{\frac{n+1}{n-1}}}{\left(k-\epsilon s^{2}\right)^{\frac{1}{n-1}}} .
$$

We must have $c_{1}=0$, that is, $\tilde{Q}=0$. We get

$$
Q(s)-s Q^{\prime}(s)=0 .
$$

Then it follows that

$$
Q(s)=Q^{\prime}(0) s .
$$

In this case, $\phi=c_{1} \sqrt{1+c_{2} s^{2}}$ where $c_{1}>0$ and $c_{2}$ are numbers independent of $s$. This case is excluded in the assumption. Therefore $k=0$ and $\epsilon=0$. Then (47) is reduced to

$$
r_{i j}=-\lambda\left(s_{j} b_{i}+s_{i} b_{j}\right)
$$

Then

$$
r_{j}+s_{j}=\left(1-\lambda b^{2}\right) s_{j}
$$

By the assumption at the beginning of the proof, $r_{j}+s_{j} \neq 0$, we conclude that $1-\lambda b^{2} \neq 0$ and $s_{j} \neq 0$. By Lemma 6.1, $\phi=\phi(s)$ satisfies (50). Let

$$
E Q:=\Delta^{2}\left\{-2 \Psi-\frac{Q \Phi}{\Delta^{2}}-\lambda\left(\frac{s \Phi}{\Delta^{2}}-2 \Psi b^{2}\right)-\delta\right\} .
$$


We have

$$
E Q=\Omega_{0}+\Omega_{2} b^{2}+\Omega_{4} b^{4}
$$

where

$$
\Omega_{4}=\left(Q^{\prime}\right)^{2}(\lambda-\delta)
$$

By $(50), E Q=0$. Thus

$$
\Omega_{0}=0, \quad \Omega_{2}=0, \quad \Omega_{4}=0 .
$$

Since $Q^{\prime} \neq 0, \Omega_{4}=0$ implies that

$$
\delta=\lambda .
$$

By a direct computation, we get

$$
\Omega_{0}+\Omega_{2} s^{2}=(1+s Q)\left\{(n+1) Q\left(Q-s Q^{\prime}\right)-Q^{\prime}+\lambda\left[n s\left(Q-s Q^{\prime}\right)-1\right]\right\} .
$$

The equations $\Omega_{0}=0$ and $\Omega_{2}=0$ imply that $\Omega_{0}+\Omega_{2} s^{2}=0$, that is,

$$
(n+1) Q\left(Q-s Q^{\prime}\right)-Q^{\prime}+\lambda\left[n s\left(Q-s Q^{\prime}\right)-1\right]=0 .
$$

We obtain

$$
Q=-\frac{\left[k_{0} n(n+1)-1\right] \lambda s \pm \sqrt{\lambda k_{0}\left(k_{0}(1+n)^{2}-1+\lambda s^{2}\right)}}{k_{0}(n+1)^{2}-1} .
$$

Plugging it into $\Omega_{2}=0$ yields

$$
k_{0} \lambda=0 .
$$

Then

$$
Q=\frac{\lambda s}{k_{0}(n+1)^{2}-1} .
$$

This implies that $\phi=k_{1} \sqrt{1+k_{2} s^{2}}$ where $k_{1}>0$ and $k_{2}$ are numbers independent of $s$. This case is excluded in the assumption of the lemma. Therefore, $r_{j}+s_{j}=0$.

Q.E.D.

Proposition 6.3 Let $F=\alpha \phi(s), s=\beta / \alpha$, be an $(\alpha, \beta)$-metric. Suppose that $\phi=\phi(s)$ satisfies (46) and $\phi \neq k_{1} \sqrt{1+k_{2} s^{2}}+k_{3} s$ for any constants $k_{1}>0, k_{2}$ and $k_{3}$. If $F$ is of isotropic S-curvature, $\mathbf{S}=(n+1) c F$, then

$$
r_{i j}=\epsilon\left(b^{2} a_{i j}-b_{i} b_{j}\right), \quad s_{j}=0,
$$

where $\epsilon=\epsilon(x)$ is a scalar function on $M$ and $\phi=\phi(s)$ satisfies

$$
\epsilon\left(b^{2}-s^{2}\right) \frac{\Phi}{2 \Delta^{2}}=-(n+1) c \phi .
$$


Proof: Contracting (47) with $b^{i}$ yields

$$
r_{j}+s_{j}=\left(k-\epsilon b^{2}\right) b_{j}+\left(1-\lambda b^{2}\right) s_{j} .
$$

By Lemma $6.2, r_{j}+s_{j}=0$. It follows from (63) that

$$
\left(1-\lambda b^{2}\right) s_{j}+\left(k-\epsilon b^{2}\right) b_{j}=0 .
$$

Contracting (64) with $b^{j}$ yields

$$
\left(k-\epsilon b^{2}\right) b^{2}=0 .
$$

We get

$$
k=\epsilon b^{2} .
$$

Then (47) is reduced to

$$
r_{i j}=\epsilon\left(b^{2} a_{i j}-b_{i} b_{j}\right)-\lambda\left(b_{i} s_{j}+b_{j} s_{i}\right) .
$$

By (49),

$$
\nu=0 .
$$

Then (48) is reduced to (62).

We claim that $s_{0}=0$. Suppose that $s_{0} \neq 0$. By (64), we conclude that

$$
\lambda=\frac{1}{b^{2}} \text {. }
$$

By (51),

$$
\delta=0 .
$$

It follows from (50) that

$$
\left(b^{2} Q+s\right) \Phi=0 .
$$

This is impossible by the assumption $\Phi \neq 0$.

Q.E.D.

\section{Proof of Theorem 1.1}

Notice that in Lemma 6.1, there is no restriction on $\phi$ other than (46). Let $\phi=k_{1} \sqrt{1+k_{2} s^{2}}+k_{3} s$ where $k_{1}>0, k_{2}$ and $k_{3}$ are numbers independent of $s$. It is easy to check that, if $k_{3} \neq 0$, then $\phi$ satisfies (46). Let $F=\alpha \phi(\beta / \alpha)$, where $\alpha$ is a Riemannian metric and $\beta$ is a 1 -form on an $n$-dimensional manifold. It is easy to see that if $F$ is a Finsler metric, then $1+k_{2} b^{2}>0$, where $b:=\left\|\beta_{x}\right\|_{\alpha}$. By Lemma 6.1, we can easily prove Theorem 1.1.

Proof of Theorem 1.1: Assume that $F$ is of isotropic S-curvature, $\mathbf{S}=(n+1) c F$. By Lemma $6.1, \beta$ satisfies (47) and $\phi$ satisfies (48) and further it satisfies (50) if $s_{0} \neq 0$.

First, we plug $\phi=k_{1} \sqrt{1+k_{2} s^{2}}+k_{3} s$ into

$$
e q:=-2 s\left(k-\epsilon b^{2}\right) \Psi+\left(k-\epsilon s^{2}\right) \frac{\Phi}{2 \Delta^{2}}+(n+1) c \phi-s \nu .
$$


By (48), the coefficients of the Taylor expansion of eq in $s$ must be zero. We obtain

$$
\begin{aligned}
c & =\frac{k_{3} k}{2\left(1+k_{2} b^{2}\right) k_{1}^{2}} \\
\nu & =\left\{\left(\frac{n}{1+k_{2} b^{2}}+1\right) \frac{k_{3}^{2}}{k_{1}^{2}}-k_{2}\right\} k \\
\epsilon & =\left\{\frac{k_{3}^{2}}{k_{1}^{2}}-k_{2}\right\} k .
\end{aligned}
$$

Assume that $s_{0} \neq 0$. We plug $\phi=k_{1} \sqrt{1+k_{2} s^{2}}+k_{3} s$ into

$$
E Q=-2 \Psi-\frac{Q \Phi}{\Delta^{2}}-\lambda\left(\frac{s \Phi}{\Delta^{2}}-2 \Psi b^{2}\right)-\delta .
$$

By (50), the coefficients of the Taylor expansion of $E Q$ in $s$ must be zero. We obtain

$$
\begin{aligned}
\lambda & =\frac{k_{3}^{2}}{k_{1}^{2}}-k_{2} \\
\delta & =\left(\frac{n}{1+k_{2} b^{2}}+1\right) \frac{k_{3}^{2}}{k_{1}^{2}}-k_{2} .
\end{aligned}
$$

This proves the necessary conditions by (47).

Conversely, if $\beta$ satisfies (4), then $F$ is of isotropic S-curvature by (18). The proof is direct, so is omitted.

Q.E.D.

\section{References}

[1] D. Bao and C. Robles, On Randers metrics of constant curvature, Rep. on Math. Phys., 51(2003), 9-42.

[2] D. Bao, C. Robles and Z. Shen, Zermelo navigation on Riemannian manifolds, J. Differential Geometry, 66(2004),377-435.

[3] D. Bao and Z. Shen, Finsler metrics of constant flag curvature on the Lie group $S^{3}$, J. London Math. Soc. 66(2002), 453-467.

[4] X. Chen, X. Mo and Z. Shen, On the flag curvature of Finsler metrics of scalar curvature, J. of London Math. Soc., 68(2)(2003),762-780.

[5] X. Chen and Z. Shen, Randers metrics with special curvature properties, Osaka J. of Math., 40(2003), 87-101.

[6] X. Cheng and Z. Shen, Projectively flat Finsler metrics with almost isotropic S-curvature, Acta Math. Scientia, 26B(2)(2006), 307-313.

[7] X. Cheng and Z. Shen, Randers metrics of scalar flag curvature, preprint, 2005 . 
[8] C.-W. Kim and J.-W. Yim, Finsler manifolds with positive constant flag curvature, Geom. Dedicata (to appear).

[9] B. Li and Z. Shen, On a class of weakly Landsberg metrics, preprint, 2006.

[10] Z. Shen, Volume compasion and its applications in Riemann-Finsler geometry, Advances in Math. 128(1997), 306-328.

[11] Z. Shen, Differential Geometry of Spray and Finsler Spaces, Kluwer Academic Publishers, 2001.

[12] Z. Shen, Finsler metrics with $K=0$ and $S=0$, Canadian J. Math. $\mathbf{5 5}(2003), 112-132$.

[13] Z. Shen, On a class of Landsberg metircs, preprint, 2006.

[14] Z. Shen, Landsberg curvature, S-curevature, and Riemann curvature, In "A Sampler of Finsler Geometry" MSRI series, Cambridge University Press, 2004 .

[15] Z. Shen, Nonpositively curved Finsler manifolds with constant S-curvature, Math. Z. 249(2005), 625-639

[16] Z. Shen, Lectures on Finsler Geometry, World Scientific Publishers, 2001.

[17] Z. Shen and H. Xing, On Randers metrics with isotropic S-curvature, Acta Math. Sinica, to appear.

[18] Z. Shen and G. C. Yildirim, Randers metrics with special flag curvature, preprint, 2005.

\author{
Xinyue Cheng \\ Department of Mathematics \\ Chongqing Institute of Technology \\ Chongqing 400050 \\ P. R. China \\ chengxy@cqit.edu.cn \\ Zhongmin Shen \\ Department of Mathematical Sciences \\ Indiana University-Purdue University Indianapolis(IUPUI) \\ 402 N. Blackford Street \\ Indianapolis, IN 46202-3216, USA \\ zshen@math.iupui.edu \\ and \\ Center of Mathematical Sciences \\ Zhejiang University \\ Hangzhou, Zhejiang Province 310027 \\ P. R. China
}

